\title{
Evaluations of Similarity Measures on VK for Link Prediction
}

\author{
JooYoung Lee ${ }^{1} \cdot$ Rustam Tukhvatov ${ }^{1}$
}

Received: 31 August 2017 / Revised: 30 March 2018 / Accepted: 3 September 2018 / Published online: 14 September 2018

(c) The Author(s) 2018

\begin{abstract}
Recommender system is one of the most important components for many companies and social networks such as Facebook and YouTube. A recommendation system consists of algorithms which allow to predict and recommend friends or products. This paper studies to facilitate finding like-minded people with same interests in social networks. In our research, we used real data from the most popular social network in Russia, VK (Vkontakte). The study is motivated on the assumption that similarity breeds connection. We evaluate well-known similarity measures in the field on our collected VK datasets and find limited performance results. The result shows that majority of users in VK tend not to add possible users with whom they have common acquaintances. We also propose a topology-based similarity measure to predict future friends. Then, we compare our results with the results of other well-known methods and discuss differences.
\end{abstract}

Keywords Recommender system $\cdot$ Graph theory $\cdot$ Link prediction $\cdot$ Similarity

\section{Introduction}

Social networks help people to interact through Internet. Nowadays social network services allow to share interests via texts, music, video, etc. Clearly, people want to get more information and new contacts as fast as possible, and the information should be relevant to their preferences.

Link recommendation has become one of the most important features in online social networks and has been an active research area $[3,8-10,14,16]$. There are wellknown examples of link recommendation such as "People You May Want to Hire" on LinkedIn, "You May Know" on Google+ and "People You May Know" on Facebook. Given the tremendous academic and practical interests in link recommendation [6], we examined existing approaches and applied recommendation engine for VK social network.

We especially focus on the most popular similarity measures which are used in the literature, namely, cosine similarity, common neighbors, Jaccard similarity and

JooYoung Lee

j.lee@innopolis.ru

Rustam Tukhvatov

r.tukhvatov@innopolis.ru

1 Network and Information Science Laboratory, Institute of Information Systems, Innopolis University, Innopolis, Russia 420500
Adamic-Adar. We show the performance of each metric using confusion matrix plus F1 measure which is the standard baselines. We examine each metric on a set of small online social networks as well as collected VK datasets.

A social network is a graph as a data structure, the users are nodes, and the users' friendships (relations) are edges [11]. We aim to understand factors which may affect the emergence of new edges and try to predict future connections in social networks. In Sect. 2, we introduce some of major existing methods on link prediction. In Sect. 3, we present existing methods which we test and compare our results with on VK datasets. In Sect. 4, we describe in detail about our experiment settings and datasets which we collected and preprocess. Section 5, then, presents how we can evaluate the results as well as the evaluation of the most well-known methods. In Sect. 6, we show our results and discuss implications. Finally, in Sect. 7, we conclude our research.

\section{Related Work}

Wu et al. [17] study understanding users' temporal behaviors in social network platforms. The evolution of social network services is driven by the interplay between users' preferences and social network structures. Authors argue that users' future preference behavior is affected by the network 
around them and the homophily effect. In [1], authors demonstrate the developed Social Poisson Factorization (SPF), a probabilistic model that incorporates social network information into a traditional factorization method. SPF introduces the social aspect of algorithmic recommendation. They develop a scalable algorithm for analyzing data with SPF, and demonstrate that it outperforms competing methods on six real-world datasets.

Zhang et al. [18] studied the social influence problem in a large microblogging network, Weibo.com. ${ }^{1}$ They investigate (re)tweet behaviors of users by considering ego network of users. In our proposed method, we expand another step by considering second level degrees of users. Kooti et al. [5] demonstrate online consumers' behavior and try to explain ways to improve ad (advertising) targeting systems. Researchers use information in emails such as purchase logs and communications between users to find patterns and their interaction. For example, authors measured the effect of gender and age which showed that a female email user is more likely to be an online shopper than an average male email user. Also the spending ability goes up with the age until age of 30 , then stabilizes in the early 60s and starts to drop afterward. Such findings help to predict future customers' behavior and make purchases more pleasant for consumers.

Measuring similarity between nodes is the main task in link prediction problem. In [15], authors constructed a new way to measure similarity between nodes based on gametheoretic interaction index. The basic form of the interaction index is built upon two solution concepts from game theory: the Shapley value and the Banzhaf index. It is also generalized to a wider class of solution concepts: Shapley value and Banzhaf index. Authors showed that using their approach, it is possible to improve existing results in link prediction and community detection problems.

In this paper, we consider link prediction problems, and more precisely, we discuss similarity measures for link prediction problems. One of the well-accepted hypotheses is that similar users will become future friends. Therefore, it is essential to measure similarity between users so that future links can be accurately predicted. As we will discuss throughout the paper, there exist many metrics to measure the similarity. Once the similarity is measured, there are many ways to predict future connections. One intuitive way is to have a threshold and predict users who have similarities greater than the given threshold. Other simple ways include predicting top $k$ users and top $1 \%$ of users. More advanced techniques include learning how much to predict using machine learning algorithms.

\footnotetext{
$\overline{1}$ The most popular Chinese microblogging service.
}

\subsection{VKontakte}

In this section, we focus on the datasets which we collected over 6 months from VK network. VK (VKontakte, meaning InContact) is the largest European online social network and social media service. According to SimilarWeb, ${ }^{2} \mathrm{VK}$ is the fifth most popular website in the world. As of January 2017, daily average audience is about 87 million visitors, ${ }^{3}$ with more than 410 million registered users. ${ }^{4}$

Any user in VK has a profile which contains various information. First and last names are mandatory fields, and other data such as birthday, city and interests are optional. Figure 1 is an example of a profile of the social network. In addition, users can share interesting information via posting on the profile wall as well as making repost from other users or groups, ${ }^{5}$ and each post may contain attachments-documents or media files.

VK has open APIs for application developments. It allows developers to access the server through requests. The APIs provide the ability to access some user information with his/ her consent, such as photographs, friends, profile and wall.

\section{Methods}

The link prediction problem is connected with network structure. In our research, we use similarity between users using information from their second level degrees.

\subsection{Problem Statement}

Given a snapshot of a social network $G^{t}=\left(V^{t}, E^{t}\right)$ where $V$ is a set of users and $E$ is a set of relationships, we aim to find a relationship $\left(v_{i}, v_{j}\right) \notin E^{t}$ and $\left(v_{i}, v_{j}\right) \in E^{t^{\prime}}$ where $t<t^{\prime}$.

The link prediction problem is the problem of predicting future connections in a network which may form in the network. Here, we briefly list some measures which we examine in this study.

Cosine Similarity $(A, B)=\cos (\theta)=\frac{f(A) f(B)}{|f(A)||f(B)|}$

\footnotetext{
${ }^{2}$ SimilarWeb Ltd. is a digital market intelligence company that provides web analytics, data analysis and business intelligence services for international corporations. It uses large data processing technologies to collect, measure, analyze and provide insights on behavioral patterns and statistics for the involvement of users from websites and mobile applications.

3 "Audience VKontakte" www.liveinternet.ru. Retrieved January 2017.

4 "List of VK users" Vk.com. Retrieved January 2017.

5 The VK group is a community where people can communicate with each other, exchanging ideas and proposals.
} 


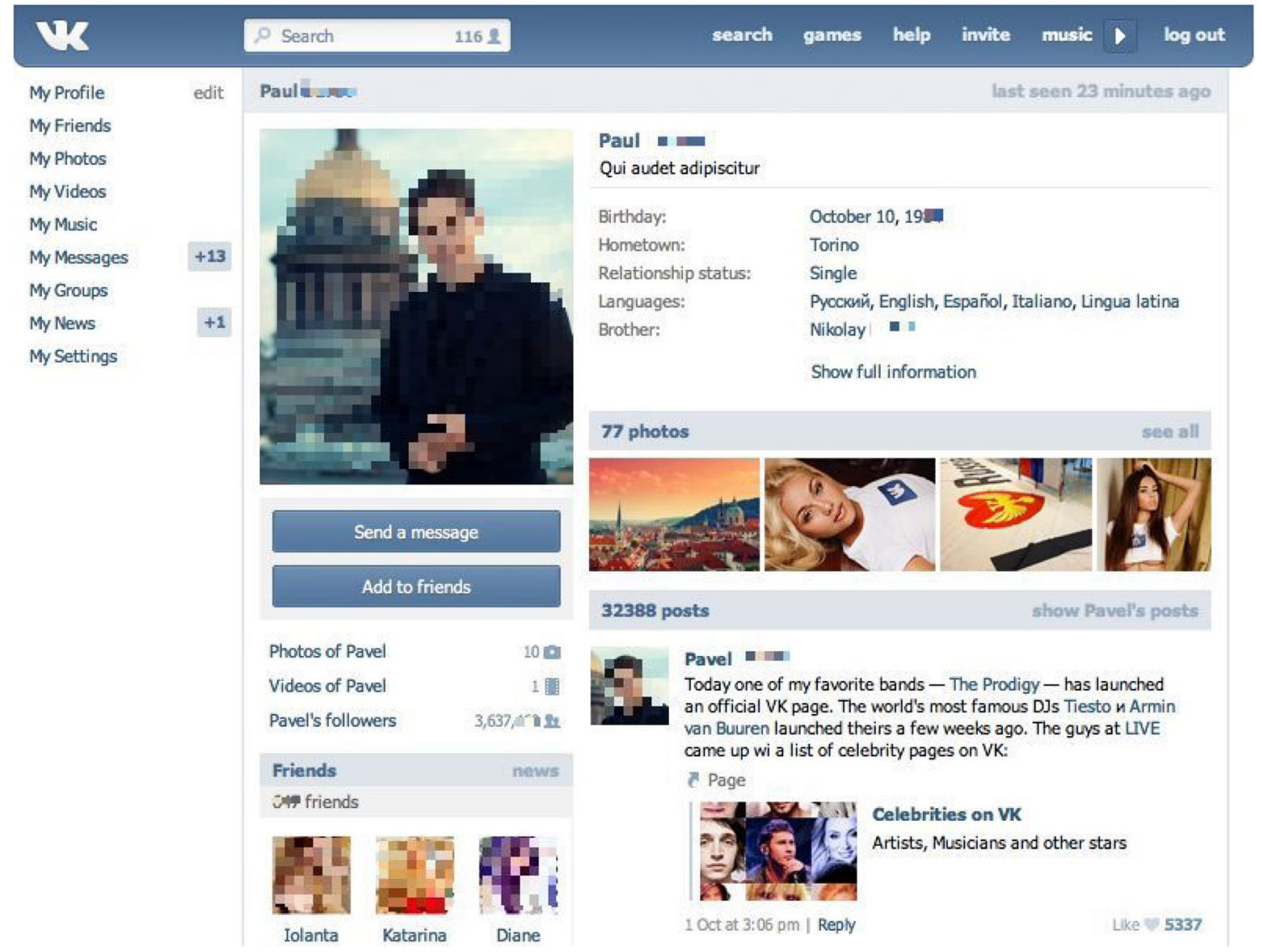

Fig. 1 VK profile

Common Neighbors $(A, B)=f(A) \cap f(b)$

Jaccard Similarity $(A, B)=\frac{|f(A) \cap f(B)|}{|f(A) \cup f(B)|}$

Adamic Adar Index $(A, B)=\sum_{w \in f(A) \cap f(B)} \frac{1}{\log |f(w)|}$

where $(f(w))$ denotes the set of neighbors of $w$.

\subsection{Second Common Neighbor Similarity}

We propose a new similarity measure between users using second degrees [7, 12]. Second neighbors are defined as the nodes which are connected in the second hops.

Second Common Neighbors $(A, B)=\left|\bigcup_{i \in f(A)} f(i) \cap \bigcup_{j \in f(B)} f(j)\right|$ where $f()$ is user's friends, $f(A)$ friend of user $A, f(i)$ is the set of neighbors of $i$.
We propose another simple metric to compare with the proposed similarity measure which is based on the shortest distance between users.

Shortest Path Index $(A, B)=$ Length of Shortest Path Between $A$ and $B$

\section{Experiments}

Ideally, the evaluation of link prediction algorithms should take place every timestamp to check the performance and adjust the algorithm if necessary. However, such an approach takes a long period of time and it is hard to track of incoming and outgoing users from the initial network. Therefore, it is common practice to delete a small portion of edges from the network as if they do not exist and then try to predict the deleted edges. This static approach has possible flaws since the similarity between users is not static; thus, deleted edges are easier to predict than future edges. 


\begin{tabular}{|c|c|c|c|c|}
\hline & id & personal & friends & wallPosts \\
\hline 0 & 2064386.0 & $\begin{array}{l}\text { [\{'uid': 2064386, 'country': } 1 \text {, 'counters': } \\
\{\text { '... }\end{array}$ & $\begin{array}{l}{[2772.0,4987.0,8680.0,11380.0,11698.0,} \\
132 \ldots\end{array}$ & $\begin{array}{l}\text { [111199386, 'Левитанский Ю.Д. <br> } \\
<\mathrm{b}>\text { Дети <b... }\end{array}$ \\
\hline 1 & 63802027.0 & $\begin{array}{l}\text { ['home_town': 'Москва', 'movies': ", } \\
\text { 'about'... }\end{array}$ & $\begin{array}{l}{[701.0,77777.0,88484.0,196957.0} \\
204655.0, \ldots\end{array}$ & $\begin{array}{l}\text { [0, 'Ура! С днем варенья, дружище! И пускай } \\
\text { вС... }\end{array}$ \\
\hline 2 & 241284438.0 & $\begin{array}{l}\text { ['home_town': ", 'movies': ", } \\
\text { 'photo_50': '... }\end{array}$ & $\begin{array}{l}{[36417.0,84922.0,125032.0,146957.0,} \\
211166 \ldots .\end{array}$ & {$[8095605, ", 4698690, ", 4247839, ", 177117 \ldots$} \\
\hline 3 & 7.0 & $\begin{array}{l}\text { [\{'uid': 7, 'counters': \{'followers': 5902, } \\
\text { 'v... }\end{array}$ & $\begin{array}{l}{[6.0,9.0,10.0,14.0,17.0,21.0,22.0,} \\
23.0, \ldots\end{array}$ & ] \\
\hline 4 & 262153.0 & $\begin{array}{l}\text { ['uid': 262153, 'country': 1, 'counters': } \\
\text { \{'u... }\end{array}$ & $\begin{array}{l}{[651.0,1174.0,3775.0,5068.0,7720.0,} \\
10437 \ldots\end{array}$ & $\begin{array}{l}\text { [0, ", -32179668, 'Новогоднее } \\
\text { печенье<br>Пече... }\end{array}$ \\
\hline
\end{tabular}

Fig. 2 Extracted data from VK

Table 1 VK networks with different time stamps

\begin{tabular}{llll}
\hline Date & Number of nodes & Number of edges & Average degree \\
\hline 23.12 .2016 & 942469 & 1475811 & 3.1318 \\
24.01 .2017 & 950503 & 1490873 & 3.1370 \\
23.02 .2017 & 936847 & 1465214 & 3.1280 \\
24.03 .2017 & 939985 & 1469854 & 3.1274 \\
\hline
\end{tabular}

In the experiment, we compare the common practice with the real link prediction based on actual future links without deleting edges. To be able to evaluate the accuracy, we collected datasets from the same set of users in different time intervals to build a learning model and then compare the predictions.

\subsection{Collection of Dataset}

We collected several snapshots of networks from VK to test link prediction methods. In order to make comparisons easier, we preprocess the data so that the resulting networks contain the same users since newly joined users in latter networks are not predictable. In the next step, we exclude users who are suspected to be not regular individuals such as groups, commercial accounts and celebrities. We removed users with more than 500 friends based on the mean number of friends in VK which was 240. Datasets contain users' profile information, friends and posts from their walls as shown in Fig. 2.
We use VK networks with four different timestamps as summarized in Table 1.

We further processed the data by removing nodes whose degree is less than two and newly joined nodes that did not exist in the starting graph. The processed networks are summarized in Table 2.

\subsection{Implementation}

We used Python programming language and developed two variants of the implementation. In the first variant, we used Pandas library to collect and process the dataset, and then, we wrote codes for the similarity measures, i.e., cosine similarity, common neighbors, Jaccard similarity and Adamic-Adar index.

In the second variant, we used Jaccard and Adamic-Adar similarity measures from Networkx library to improve the speed of our implementation.

- Python 3.5.2

Python is a high-level general-purpose programming language, focused on improving developer productivity and code readability. The syntax of the Python kernel is minimal, and at the same time, the standard library includes a large amount of useful functions.

- IPython 5.1.0

IPython is an interactive computational environment for the Python programming language, in Jupyter Note-
Table 2 VK networks after preprocessing

\begin{tabular}{llllc}
\hline Date & Number of nodes & Number of edges & Average degree & Excess nodes \\
\hline 23.12 .2016 & 190,550 & 712,014 & 7.4733 & 7412 \\
24.01 .2017 & 190,550 & 716,478 & 7.5201 & 5459 \\
23.02 .2017 & 190,550 & 712,196 & 7.4752 & 1083 \\
24.03 .2017 & 190,550 & 713,030 & 7.4839 & 340 \\
\hline
\end{tabular}


book 4.3.1 shell. It is an open-source program and one of the popular tools for data scientists.

\subsubsection{Python Libraries}

- Pandas

Pandas library makes Python a powerful tool for data analysis. It is an open-source, easy-to-use, BSD-licensed. The package allows to build summary tables, performs a grouping, provides easy access to tabular data and has robust IO tools for loading and saving data from flat files (CSV and delimited). The library is well suited for different kinds of data: forms of statistical or observational datasets, arbitrary matrix data with row and column labels, and it might be heterogeneous or homogeneously typed, tabular data like Excel spreadsheet. Pandas package provides high performance because many low-level algorithms are tweaked in Cython code. Cython language is a superset of Python that allows the compiler to generate very efficient $\mathrm{C}$ codes from Python.

- Matplotlib

Matplotlib is a plotting library for the Python, which produces publication quality figures in a variety of formats. It allows to draw graphics, plots, histograms, power spectra, bar charts, error charts and scatterplots on the resulting data sets.

- Scikit-learn

Scikit-learn is a free, BSD-licensed machine learning library for Python. It features various classification, regression and clustering algorithms including support vector machines and random forests. The library is built on $S$ ciPy (fundamental library for scientific computing and other useful tools) and NumPy (base $n$-dimensional array package)

- Networkx

NetworkX is a free, BSD-licensed library for the creation, manipulation and study of the structure, dynamics and functions of graphs and complex networks in Python.

\section{Evaluations}

To evaluate the quality of predictions, we use commonly accepted measures in data mining: precision, recall, accuracy and F1 score.

- Precision (7) is the ratio of a number of events that are correctly predicted to a number of all predicted events.

Precision $=\frac{\mathrm{tp}}{\mathrm{tp}+\mathrm{fp}}$

- Recall (8) is the ratio of a number of the events that are correctly predicted to a number of all correct events.

$$
\text { Recall }=\frac{\mathrm{tp}}{\mathrm{tp}+\mathrm{fn}}
$$

- Accuracy (9) is the ratio of correctly predicted events. Perhaps, it is the most intuitive performance measure, but it is only good for symmetric datasets where false positives and false negatives are roughly the same.

Accuracy $=\frac{\mathrm{tp}+\mathrm{tn}}{N}$

where $N(10)$ is sum of rates:

$$
N=\mathrm{fn}+\mathrm{fp}+\mathrm{tn}+\mathrm{tp}
$$

- $F_{1}$ score (11) is harmonic average of precision and recall.

$$
F_{1} \text { score }=2 \times \frac{\text { Precision } \times \text { Recall }}{\text { Precision }+ \text { Recall }}
$$

The statistical measures of the performance of a binary classification test are also called rates:

- true positives (tp) are the categories that we expected to see and received at the output of the classifier;

- false negatives (fn) are the categories, which we expected to see, but the classifier did not define them, also known type II error;

- false positives (fp) are the categories, which should not be output, but the classifier returned them erroneously at the output, also known type I error ;

- true negatives (tn) are the categories, which should not be output, and they are also correctly absent at the output of the classifier.

\subsection{Evaluation of Existing Methods}

To have a base line, we started from the most commonly used measures of similarity [13] which were introduced in the previous section, cosine (1) similarity, common neighbors (2), Jaccard (3) similarity and Adamic-Adar (4).

\subsubsection{Evaluation of Existing Methods on Facebook}

To make evaluations, we used Facebook dataset ${ }^{6}$ which contains 4039 nodes and 88,234 edges. To predict connections, $30 \%$ of the edges were deleted and after deleting, the number of edges became 61,764 . One of the questions that we need to answer is how many edges we should predict? The first approach we tried is that the similarity index (cosine, Jaccard and Adamic-Adar) should be greater than zero which led to very poor results. Then, we added a threshold value which decides if a future link should be predicted or not.

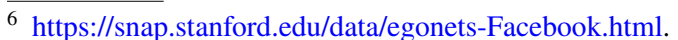




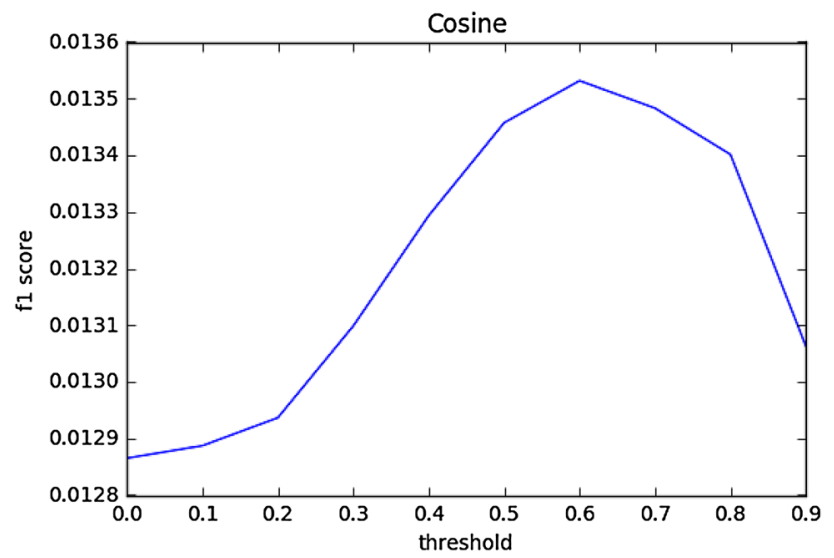

Fig. 3 Cosine similarity measure for Facebook

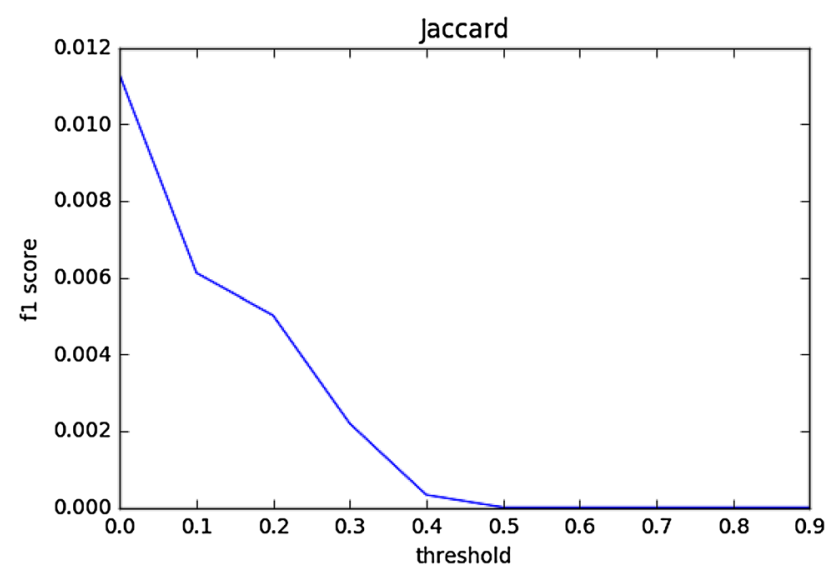

Fig. 4 Jaccard similarity measure for Facebook

The similarity measures have differences in their boundary values. The minimum value for all measures is 0 ; however, right side boundary of cosine and Jaccard is 1, whereas the right side boundary of Adamic-Adar is a float and depends on the number of intersected features. In our case, common neighbor similarity shows how many friends intersected between two users' friends lists, and therefore, the threshold is 0 . The threshold value is chosen in the range from 0 to 1 . The results of the threshold determination are represented in Figs. 3, 4 and 5. The influence of the threshold value is compared to F1-score.

After the previous evaluations, the thresholds for Jaccard and Adamic-Adar were not useful since the thresholds were equal to 0 and for cosine measure, best score is shown when the threshold is 0.6. The question "how many edges we should predict?" is very difficult to answer, and the performance depends on the answer. Table 3 shows the evaluation results of similarity measures using precision, recall, accuracy and F1-score.

Analyzing the results, we might say that common neighbor and Jaccard similarity measures show the same values.

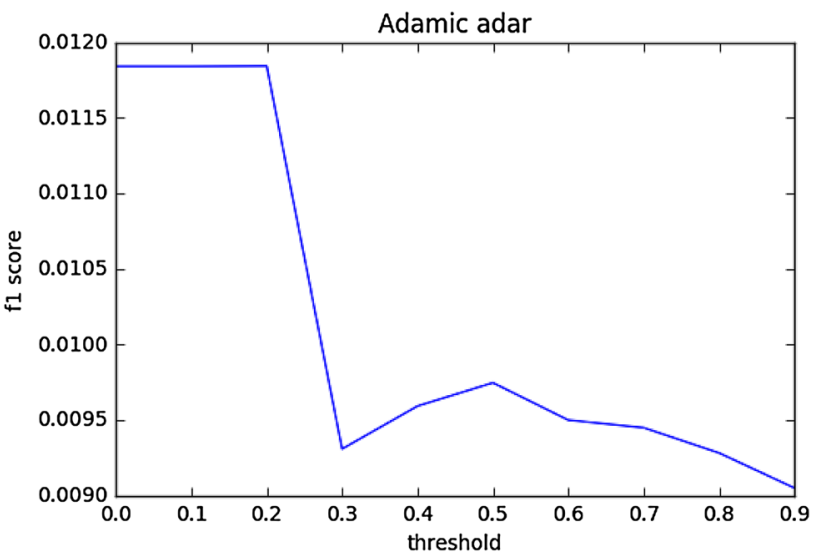

Fig. 5 Adamic-Adar similarity measure for Facebook

Table 3 Evaluation measures for Facebook dataset

\begin{tabular}{lllll}
\hline & Common neighbor & Cosine & Jaccard & Adamic-Adar \\
\hline Precision & 0.009341 & 0.006901 & 0.009341 & $\mathbf{0 . 0 1 0 1 0 8}$ \\
Recall & $\mathbf{0 . 0 4 0 1 3 0}$ & 0.346467 & $\mathbf{0 . 0 4 0 1 3 0}$ & 0.037982 \\
Accuracy & 0.966173 & 0.746648 & 0.966173 & $\mathbf{0 . 9 6 9 7 0 9}$ \\
F1 score & 0.015155 & 0.013532 & 0.015155 & $\mathbf{0 . 0 1 5 9 6 7}$ \\
\hline
\end{tabular}

Bold values indicate the best or the worst results

Adamic-Adar index shows the highest precision, accuracy and F1-score values in this example. Accuracy demonstrates high values, the reason is that TN in the is big, and accuracy contains $\mathrm{TN}$ in the definition. In our case, true negatives denote those connections that we did not predict and they did not really happen. Let us assume that we have a graph with 1000 nodes and 15,000 edges. The graph is not complete. Number of edges for complete graph can be found from formula:

Number of Edges $=\frac{n(n-1)}{2}$

where $n$ is number of nodes.

So, for graph with 1000 nodes, number of possible edges is equal to 499,500 . If we predict 5000 future links (new edges), true positive is equal:

$\mathrm{TP}=\mathrm{APL}-\mathrm{EL}-\mathrm{PrL}$

where APL is number of all possible links,

EL is number of existed links,

PrL is number of predicted links

$\mathrm{TP}=499,500-15,000-5000=479,500$, so now it is clear why TN is such big. Therefore, further accuracy does not greatly affect our analysis.

To examine popular similarity measures to predict links, we used data from Vkontakte (VK) social network. First, we processed Facebook dataset. The average number of users' 
Table 4 Evaluating measures table for VK (baseline)

\begin{tabular}{lllll}
\hline & Common neighbor & Cosine & Jaccard & Adamic-Adar \\
\hline Precision & 0.002936 & $\mathbf{0 . 0 1 9 1 2 1}$ & 0.002936 & 0.014459 \\
Recall & 0.008744 & $\mathbf{0 . 1 7 1 4 3 2}$ & 0.008744 & 0.005208 \\
Accuracy & 0.837793 & $\mathbf{0 . 9 5 9 5 8 9}$ & 0.837793 & 0.822696 \\
F1 score & 0.004395 & 0.0005134 & 0.004395 & $\mathbf{0 . 0 0 7 6 5 8}$ \\
\hline
\end{tabular}

Bold values indicate the best or the worst results

Table 5 Datasets

\begin{tabular}{lrrr}
\hline Dataset & Nodes & Edges & Avg. degree \\
\hline Facebook-1 & 324 & 2218 & 13.6914 \\
Facebook-1_del & 320 & 1553 & 9.7063 \\
Last.fm & 1892 & 12,717 & 13.4429 \\
Last.fm_del & 1801 & 8902 & 9.8856 \\
GrQc & 5242 & 14,496 & 5.5307 \\
GrQc_del & 4729 & 10,147 & 4.2914 \\
HepTh & 9877 & 25,998 & 5.2644 \\
HepTh_del & 9020 & 18,199 & 4.0353 \\
CondMat & 23133 & 93,497 & 8.0834 \\
CondMat_del & 21982 & 65,448 & 5.9547 \\
\hline
\end{tabular}

friends is 23 in Facebook dataset and 246 in VK dataset. To efficiently process the big data, we have reduced from 10,728 nodes $(2,332,068$ edges) to 4000 user so that it contains 4000 nodes and 1,122,226 edges. To test the common practice, we deleted $30 \%$ edges and then try to predict the deleted ones. After deleting $30 \%$ of the edges, the number of edges was 784,499.

The threshold value is chosen to maximize the accuracy: 0.6 for cosine and 0 for others similarity measures. Table 4 illustrates the performance of the most popular similarity measures: precision, recall, accuracy and F1-score.

As shown in the table, the quality of predictions for VK network is lower than for Facebook dataset. One of the reasons is that VK network is very sparse and the relationships are concentrated in small regions. The results of the VK dataset show that Adamic-Adar similarity index is the best evaluating measure according to F1-score and cosine for others.

\subsection{Evaluation of Existing Methods on Different Datasets}

To further examine the similarity measures for link prediction, we used six different datasets shown in Table 5. To evaluate predictions was deleted $30 \%$ of the edges. Table 5 summarizes the original dataset and the dataset with deleted edges.

Tables $6,7,8,9,10,11,12,13,14$ and 15 illustrate performance of predictions.
Table 6 Illustration of prediction rates for the small dataset from Facebook

Cosine

\begin{tabular}{lr} 
(a) Cosine rates & \\
tp & 102 \\
fn & 22 \\
tn & 8337 \\
fp & 60,167 \\
\hline
\end{tabular}

\begin{tabular}{lr} 
(b) Jaccard rates & \\
tp & 89 \\
fn & 35 \\
tn & 56,306 \\
$\mathrm{fp}$ & 9504 \\
\hline & Common \\
\hline (c) Common neighbor rates & \\
tp & 89 \\
fn & 35 \\
tn & 56,306 \\
fp & 9504 \\
\hline & Adamic \\
\hline (d) Adamic-Adar rates & 59 \\
tp & 65 \\
fn & 58,243 \\
tn & 74,874 \\
fp &
\end{tabular}

Table 7 Evaluating similarity measures for the small dataset from Facebook

\begin{tabular}{lllll}
\hline & Precision & Recall & Accuracy & F1-score \\
\hline Cosine & 0.001692 & 0.822581 & 0.122967 & 0.003378 \\
Jaccard & 0.009278 & 0.717742 & 0.855325 & 0.018318 \\
Common & 0.009278 & 0.717742 & 0.855325 & 0.018318 \\
Adamic & 0.007819 & 0.475806 & 0.885322 & 0.015385 \\
\hline
\end{tabular}

\subsection{A Small Dataset from Facebook}

This Facebook dataset differs from the Facebook data which we used in Sect. 5.1.1. This is the smallest dataset among the presented data in Table 5 with the biggest average degrees. Table 6 illustrates the confusion matrices for the small dataset from Facebook where all measures give plenty false alarms. The best TP result is from cosine similarity. Jaccard and common neighbor similarity measures demonstrate the same results in all rates. According to Table 7, the best recall is from cosine similarity but at the same time, the worst results in precision which leads to the worst F1-score. 


\subsection{LastFM}

This dataset contains a social network from LastFM social networking platform. Table 8 illustrates the confusion matrices for LastFM dataset where all measures give plenty of false alarms. The best TP result is from cosine similarity. Jaccard and common neighbor similarity measures demonstrate the same results in all prediction rates. According to Table 9 , the best recall result is from cosine similarity with the worst results in precision which leads to the worst F1-score.

Table 8 Illustration of prediction rates of LastFM dataset

\begin{tabular}{lr}
\hline & \multicolumn{1}{c}{ Cosine } \\
\hline (a) Cosine rates & 406 \\
tp & 168 \\
fn & 384,640 \\
tn & $1,424,806$ \\
fp & Jaccard \\
\hline
\end{tabular}

\begin{tabular}{lr} 
(b) Jaccard rates & \\
tp & 176 \\
$\mathrm{fn}$ & 398 \\
$\mathrm{tn}$ & $1,569,014$ \\
$\mathrm{fp}$ & 124,157 \\
\hline & Common
\end{tabular}

\begin{tabular}{lr}
\hline (c) Common neighbor rates & \\
tp & 176 \\
$\mathrm{fn}$ & 398 \\
$\mathrm{tn}$ & $1,569,014$ \\
$\mathrm{fp}$ & 124,157 \\
\hline & Adamic
\end{tabular}

\begin{tabular}{lr}
\hline (d) Adamic-Adar rates & \\
tp & 136 \\
$\mathrm{fn}$ & 438 \\
$\mathrm{tn}$ & $1,588,450$ \\
$\mathrm{fp}$ & 104,017 \\
\hline
\end{tabular}

Table 9 Evaluating similarity measures for LastFM dataset

\begin{tabular}{lllll}
\hline & Precision & Recall & Accuracy & F1-score \\
\hline Cosine & 0.000285 & 0.707317 & 0.212730 & 0.000570 \\
Jaccard & 0.001416 & 0.306620 & 0.926462 & 0.002818 \\
Common & 0.001416 & 0.306620 & 0.926462 & 0.002818 \\
Adamic & 0.001306 & 0.236934 & 0.938303 & 0.002597 \\
\hline
\end{tabular}

\subsection{GrQc}

General relativity and quantum cosmology (GR-QC) collaboration network is from the e-print arXiv and covers scientific collaborations among authors with papers submitted to General Relativity and Quantum Cosmology category.

Table 10 illustrates the confusion matrix for GrQc dataset. As shown in the table, again, all measures are prone to false alarms. According to Table 11, the best recall is given by cosine similarity and the worst results in precision as well as F1-score.

Table 10 Illustration of similarity rates for GrQc dataset

\begin{tabular}{|c|c|}
\hline & Cosine \\
\hline \multicolumn{2}{|c|}{ (a) Cosine rates } \\
\hline tp & 472 \\
\hline fn & 479 \\
\hline tn & $3,018,531$ \\
\hline \multirow[t]{2}{*}{ fp } & $5,461,423$ \\
\hline & Jaccard \\
\hline \multicolumn{2}{|c|}{ (b) Jaccard rates } \\
\hline tp & 321 \\
\hline fn & 630 \\
\hline tn & $7,853,685$ \\
\hline \multirow[t]{2}{*}{$\mathrm{fp}$} & 29,385 \\
\hline & Common \\
\hline \multicolumn{2}{|c|}{ (c) Common neighbor rates } \\
\hline tp & 321 \\
\hline fn & 630 \\
\hline tn & $7,853,685$ \\
\hline \multirow[t]{2}{*}{ fp } & 29,385 \\
\hline & Adamic \\
\hline \multicolumn{2}{|c|}{ (d) Adamic-Adar rates } \\
\hline tp & 147 \\
\hline fn & 804 \\
\hline tn & $7,861,336$ \\
\hline $\mathrm{fp}$ & 21,129 \\
\hline
\end{tabular}

Table 11 Evaluating measures table for GrQc dataset

\begin{tabular}{lllll}
\hline & Precision & Recall & Accuracy & F1-score \\
\hline Cosine & 0.000086 & 0.496320 & 0.355977 & 0.000173 \\
Jaccard & 0.010806 & 0.337539 & 0.996193 & 0.020941 \\
Common & 0.010806 & 0.337539 & 0.996193 & 0.020941 \\
Adamic & 0.006909 & 0.154574 & 0.997218 & 0.013227 \\
\hline
\end{tabular}




\subsection{HepTh}

High energy physics theory (HEP-TH) citation graph is from the e-print arXiv. The average degree of this dataset is the smallest among all the presented datasets in Table 5. Table 12 illustrates the confusion matrices for HepTh dataset. According to Table 13, the best recall is given by cosine similarity.

\subsection{CondMat}

Condense matter physics (CondMat) collaboration network is from the e-print arXiv and covers scientific collaborations between papers submitted to Condense Matter category. This

Table 12 Illustration of various similarity rates for HepTh dataset

\begin{tabular}{lr}
\hline & \multicolumn{1}{c}{ Cosine } \\
\hline (a) Cosine rates & \\
tp & 1073 \\
fn & 869 \\
tn & $10,804,371$ \\
fp & $21,680,233$ \\
\hline & Jaccard \\
\hline b) Jaccard rates & 681 \\
tp & 1261 \\
fn & 82,874 \\
tn & Common \\
fp & $29,960,801$ \\
\hline &
\end{tabular}

(c) Common neighbor rates

Common

tp

fn

tn

fp

Adamic

tp

fn

tn

fp

53,063 (d) Adamic-Adar rates

is the biggest dataset among the presented dataset in Table 5. Table 14 illustrates the confusion matrices for CondMat dataset. According to Table 15, the best recall is given by cosine similarity.

Table 14 Illustration of similarity rates for CondMat dataset

\begin{tabular}{lr}
\hline & \multicolumn{1}{c}{ Cosine } \\
\hline (a) Cosine rates & \\
tp & 3817 \\
fn & 2323 \\
tn & $59,017,890$ \\
$\mathrm{fp}$ & $190,840,644$ \\
\hline
\end{tabular}

(b) Jaccard rates

tp 2820

fn $\quad 3320$

tn $\quad 230,958,602$

\begin{tabular}{ll}
$\mathrm{fp}$ & 627,368 \\
\hline
\end{tabular}

Common

(c) Common neighbor rates

tp 2820

fn $\quad 3320$

tn $\quad 230,958,602$

fp $\quad 627,368$

Adamic

(d) Adamic-Adar rates

tp 1610

fn $\quad 4530$

th $231,080,071$

fp $\quad 499,409$

Table 15 Evaluating measures table for CondMat dataset

\begin{tabular}{lllll}
\hline & Precision & Recall & Accuracy & F1-score \\
\hline Cosine & 0.000020 & 0.621661 & 0.236215 & 0.000040 \\
Jaccard & 0.004475 & 0.459283 & 0.997277 & 0.008863 \\
Common & 0.004475 & 0.459283 & 0.997277 & 0.008863 \\
Adamic & 0.003213 & 0.262215 & 0.997824 & 0.006349 \\
\hline
\end{tabular}

Table 16 Evaluating measures table for VK (deleting approach)

Table 13 Evaluating measures table for HepTh dataset

\begin{tabular}{lllll}
\hline & Precision & Recall & Accuracy & F1-score \\
\hline Cosine & 0.000049 & 0.552523 & 0.332613 & 0.000099 \\
Jaccard & 0.008150 & 0.350669 & 0.997200 & 0.015930 \\
Common & 0.008150 & 0.350669 & 0.997200 & 0.015930 \\
Adamic & 0.005976 & 0.164264 & 0.998180 & 0.011532 \\
\hline
\end{tabular}

\begin{tabular}{llll}
\hline & Cosine & Jaccard & Adamic-Adar \\
\hline Precision & 0.0004111 & 0.0053608 & 0.0289651 \\
Recall & 0.0428219 & 0.0403065 & 0.0016984 \\
Accuracy & 0.0085148 & 0.9190919 & 0.8309117 \\
F1 score & 0.0008144 & $\mathbf{0 . 0 0 9 4 6 2 9}$ & 0.0032087 \\
\hline
\end{tabular}

Bold value indicates the best or the worst result 
Based on the performance results that we showed for five different datasets, we can observe how different measures behave on different datasets. Generally, what we want to have is high TP and low FP. In reality, however, when TP grows FP also grows. The same applies to TN and FN. Therefore, at least for these five datasets that we examined here, cosine similarity is not a good metric for link prediction problems since it results in high false positives. Although it has the highest TP rate among the four similarity measures under examination, it is only at the cost of high FP. We carefully suggest that, according to the results, Jaccard similarity is the most successful measure among the four, since it stably shows high TP rate with low FP as well as high TN and low FN. Adamic-Adar similarity sometimes has better performance than Jaccard similarity, but it is not as stable since sometimes it shows poor TP rate.

\section{Results}

Obtained results implies that it is possible to predict future connections at the price of false positives. Also the increasing size of a network (number of nodes) implies the exponential increase of TN. To overcome such shortcomings, we improvised new approaches.

\subsection{Next Steps of Prediction on VK}

The next step of our predictions using VK dataset was the assumption that not all user are "useful" and we should exclude them from our dataset. The assumption of "Usefulness" concerns that some profiles are not profiles of an ordinary user, but a company profile or a famous person (like singers), some profiles are not may be companies or popular people and in both cases they do not need to have recommendations otherwise the mean and the median of users in our dataset are about 240 friends also based on Dunbar's number [2]; therefore, we delete users who have more than 500 users in their friend list.

The dataset contains 13,951 users, after excluding users who have more than 500 friends. To make predictions, we deleted $30 \%$ of the edges.

Table 16 illustrates evaluations of similarity measures: precision, recall, accuracy and F1-score. We found that Jaccard similarity has the best values in precision and F1-score.

\subsubsection{Wall Analysis}

As described in Sect. 2.1, any user of the VK social network may repost some information and these posts are attached to his wall. The posts have id of user or group, which shows
Table 17 Evaluating measures table for VK (real data comparisons)

\begin{tabular}{llll}
\hline & Cosine & Jaccard & Adamic-Adar \\
\hline Precision & 0.0000038 & 0.0000297 & 0.0001549 \\
Recall & 0.0001232 & 0.0001102 & 0.0000779 \\
Accuracy & 0.0030727 & 0.8596805 & 0.9532686 \\
F1 score & 0.0000073 & 0.0000468 & 0.0001037 \\
\hline
\end{tabular}

where the repost was made from. Combining gathered frequency of posts and reposted places (ids where the repost was made from), we introduced a similarity measure of user $\mathrm{A}$ and user B:

Wall Similarity $=\log (I(A)+R(B))$

where $I$ (shared ids) is number of common groups of two users

where $R$ (shared reposts) is number of common posts of two users

\subsubsection{Real Data}

We compare VK dataset with 6966 users which was collected on December 22, 2016, with 1,506,714 edges and the dataset with the same users which was collected on January 24, 2017, which contains 1,523,040 edges. Table 17 illustrates the obtained results.

\subsection{Proposed Measure}

In this section, we present the new approaches to overcome the shortcomings shown before.

1. According to the evaluation further we pay attention only to TP, FP and FN rates, without using precision, recall, F1-score and accuracy.

2. Also we try to limit the number of predictions and select only the highest values of similarity to predict future connections.

3. In Sect. 6.1.1, we describe results of the approach using users' walls to find similarity between users.

\subsection{Evaluation of Other Approaches}

Tables 18, 19 and 20 illustrate results of the similarity measures when we limited the number of predictions by selecting the top $2 \%$ from the best similarity indexes for 1000 randomly selected users.

We omit common neighbor similarity here since it shows similar results with Jaccard index and cosine. Instead we add two other measures, i.e., second neighbor similarity in Eq. (5) and shortest path length in Eq. (6). 
Table 18 Illustration of various rates (data from January)

Jaccard

(a) Jaccard rates

\begin{tabular}{lc}
$\mathrm{tp}$ & 0 \\
$\mathrm{fn}$ & 534 \\
$\mathrm{fp}$ & 1006 \\
\hline
\end{tabular}

\begin{tabular}{lr}
\hline (b) Adamic-Adar rates & \\
tp & 0 \\
$\mathrm{fn}$ & 534 \\
$\mathrm{fp}$ & 1006 \\
\hline
\end{tabular}

Second

(c) Second neighbor rates

\begin{tabular}{lr}
$\mathrm{tp}$ & 0 \\
$\mathrm{fn}$ & 4608 \\
$\mathrm{fp}$ & 1006 \\
\hline & Second \\
\hline (d) Shortest path rates & \\
$\mathrm{tp}$ & 0 \\
$\mathrm{fn}$ & 4800 \\
$\mathrm{fp}$ & 1006
\end{tabular}

Table 19 Illustration of various rates (data from February 2017)

Jaccard

Jaccard

\begin{tabular}{lr}
\hline (a) Jaccard rates & \\
$\mathrm{tp}$ & 0 \\
$\mathrm{fn}$ & 534 \\
$\mathrm{fp}$ & 1252 \\
\hline
\end{tabular}

\begin{tabular}{lc}
\hline (b) Adamic-Adar rates & \\
$\mathrm{tp}$ & 0 \\
$\mathrm{fn}$ & 534 \\
$\mathrm{fp}$ & 1252 \\
\hline & Second
\end{tabular}

\begin{tabular}{lc}
\hline (c) Second neighbor rates & \\
$\mathrm{tp}$ & 0 \\
$\mathrm{fn}$ & 4608 \\
$\mathrm{fp}$ & 1252 \\
\hline & Shortest
\end{tabular}

(d) Shortest path rates

$\begin{array}{rr}\mathrm{tp} & 0 \\ \mathrm{fn} & 4800 \\ \mathrm{fp} & 1252\end{array}$

The main outcome of the results is that TP is equal to zero which implies that we predict very small number of potential relationships.

\subsubsection{Top 25}

In this experiment, we select $25 \%$ from the best similarity indexes for 1000 randomly selected users. Results are summarized in Tables 21, 22 and 23. The values for TP have increased, but we still have big false predictions.

\subsection{Comparison of Second Neighbor Approach}

In this section, we have used Facebook dataset which is previously presented. We have deleted $30 \%$ edges and then make prediction for 1000 randomly selected users. Table 24 illustrates that the proposed second neighbor measure shows the best TP and lowest FP with smaller FN compared to the shortest path measure.

\section{Conclusion and Future Work}

There exist many different types of link prediction approaches, and they can be roughly categorized into learning-based methods and proximity-based methods. In our research, we considered proximity-based methods, namely, cosine similarity, common neighbor, Jaccard similarity, Adamic-Adar index, second neighbor and shortest path. All of them are structural proximity-based methods which does not consider nodal information. We have thoroughly tested the measures on different datasets. Many existing studies make prediction only for randomly deleted edges due to lack of real datasets. We collected our own snapshots of graphs from VK social network within 4 months. To facilitate the collected dataset for prediction, we filtered users that have too many links and also maintained the same users for each snapshot since we predict links but not new users in this study.

The results do not conclude which approach is superior in most of the cases. The prediction method to use for real networks should be chosen with heuristics given the nature of the network. In this study, we showed that there is a great difference with the same measures in VK and Facebook datasets.

In [4], authors discuss on the problem of current link prediction approaches. We have pointed out that evaluating link predictions based on deleted edges is not suitable considering the dynamics of social networks. In addition, links are not only added but also deleted over time. Since this fact also affects the topological similarity measures, it is not effective to predict future links only based on the current snapshot of the network. We plan to work 
Table 20 Illustration of various rates (data from March 2017)

\begin{tabular}{lr}
\hline & Jaccard \\
\hline (a) Jaccard rates & \\
$\mathrm{tp}$ & 0 \\
$\mathrm{fn}$ & 534 \\
$\mathrm{fp}$ & 1567 \\
\hline & Adamic
\end{tabular}

\begin{tabular}{lr}
\hline (b) Adamic-Adar rates & \\
$\mathrm{tp}$ & 0 \\
$\mathrm{fn}$ & 534 \\
$\mathrm{fp}$ & 1567 \\
\hline
\end{tabular}

\begin{tabular}{lc}
\hline (c) Second neighbor rates & \\
$\mathrm{tp}$ & 0 \\
$\mathrm{fn}$ & 4608 \\
$\mathrm{fp}$ & 1567 \\
\hline & Shortest
\end{tabular}

\begin{tabular}{lr}
\hline (d) Shortest path rates & \\
tp & 0 \\
fn & 4800 \\
$\mathrm{fp}$ & 1567 \\
\hline
\end{tabular}

Table 21 Illustration of various rates (data from January 2017)

\begin{tabular}{lr}
\hline & Jaccard \\
\hline (a) Jaccard rates & \\
tp & 2 \\
$\mathrm{fn}$ & 24,336 \\
$\mathrm{fp}$ & 397 \\
\hline
\end{tabular}

\begin{tabular}{lr}
\hline (b) Adamic-Adar rates & \\
$\mathrm{tp}$ & 2 \\
$\mathrm{fn}$ & 24,336 \\
$\mathrm{fp}$ & 397 \\
\hline
\end{tabular}

\begin{tabular}{lr} 
(c) Second Neighbor rates & \\
$\mathrm{tp}$ & 2 \\
$\mathrm{fn}$ & 211,130 \\
$\mathrm{fp}$ & 397 \\
\hline
\end{tabular}

\begin{tabular}{lr}
\hline (d) Shortest path rates & \\
tp & 2 \\
fn & 219,836 \\
fp & 397 \\
\hline
\end{tabular}

Table 22 Illustration of various rates (data from February 2017)

Jaccard

\begin{tabular}{lr} 
(a) Jaccard rates & \\
tp & 3 \\
$\mathrm{fn}$ & 24,335 \\
$\mathrm{fp}$ & 459 \\
\hline
\end{tabular}

(b) Adamic-Adar rates

tp 3

fn 24,335

fp $\quad 459$

Second

(c) Second neighbor rates

\begin{tabular}{lr} 
tp & 3 \\
$\mathrm{fn}$ & 211,129 \\
$\mathrm{fp}$ & 459 \\
\hline & Shortest \\
\hline (d) Shortest path rates & \\
$\mathrm{tp}$ & 3 \\
$\mathrm{fn}$ & 219,835 \\
$\mathrm{fp}$ & 459
\end{tabular}

Table 23 Illustration of various rates for $25 \%$ top predictions (data from March 2017)

Jaccard

\begin{tabular}{lr}
\hline (a) Jaccard rates & \\
$\mathrm{tp}$ & 4 \\
$\mathrm{fn}$ & 24,334 \\
$\mathrm{fp}$ & 535 \\
\hline
\end{tabular}

(b) Adamic-Adar rates

tp

4

fn $\quad 24,334$

fp

535

Second

(c) Second neighbor rates

tp

4

fn

211,128

fp

535

Shortest

(d) Shortest path rates

tp

fn

219,834

fp 
Table 24 Illustration of various rates (data from the Facebook dataset)

\begin{tabular}{lr}
\hline & Jaccard \\
\hline (a) Jaccard rates & \\
tp & 1776 \\
$\mathrm{fn}$ & 105,520 \\
$\mathrm{fp}$ & 10,212 \\
\hline & Adamic \\
\hline (b) Adamic-Adar rates & \\
$\mathrm{tp}$ & 1776 \\
$\mathrm{fn}$ & 105,520 \\
$\mathrm{fp}$ & 10,212 \\
\hline & Second \\
\hline (c) Second neighbor rates & $\mathbf{1 7 9 1}$ \\
$\mathrm{tp}$ & 545,865 \\
$\mathrm{fn}$ & $\mathbf{1 0 , 1 9 7}$ \\
$\mathrm{fp}$ & Shortest \\
\hline & \\
\hline (d) Shortest path rates & 1791 \\
$\mathrm{tp}$ & 922,919 \\
$\mathrm{fn}$ & 10,197 \\
$\mathrm{fp}$ & \\
\hline
\end{tabular}

Bold values indicate the best or the worst results

toward dynamics of networks to predict links with less false positives.

Open Access This article is distributed under the terms of the Creative Commons Attribution 4.0 International License (http://creativeco mmons.org/licenses/by/4.0/), which permits unrestricted use, distribution, and reproduction in any medium, provided you give appropriate credit to the original author(s) and the source, provide a link to the Creative Commons license, and indicate if changes were made.

\section{References}

1. Chaney AJB, Blei DM, Eliassi-Rad T (2015) A probabilistic model for using social networks in personalized item recommendation. In: Proceedings of the 9th ACM conference on recommender systems, RecSys 2015, Vienna, Austria, Sept 16-20, pp $43-50$

2. Dunbar RIM (1992) Neocortex size as a constraint on group size in primates. J Hum Evolut 22(6):469-493
3. Hussain R, Nawaz W, Lee JY, Son J, Seo JT (2016) A hybrid trust management framework for vehicular social networks. In: International conference on computational social networks. Springer, pp 214-225

4. Junuthula RR, Xu KS, Devabhaktuni VK (2016) Evaluating link prediction accuracy in dynamic networks with added and removed edges. In: 2016 IEEE international conferences on big data and cloud computing (BDCloud), social computing and networking (SocialCom), sustainable computing and communications (SustainCom) (BDCloud-SocialCom-SustainCom), pp 377-384

5. Kooti F, Lerman K, Aiello LM, Grbovic M, Djuric N, Radosavljevic V (2015) Portrait of an online shopper: understanding and predicting consumer behavior. CoRR, abs/1512.04912

6. Lebedev A, Lee JY, Rivera V, Mazzara M (2017) Link prediction using top-k shortest distances, LNCS. Springer

7. Lee JY, Oh JC (2017) Agent perspective social networks: distributed second degree estimation. Encyclopedia of social network analysis and mining, pp 1-12

8. Lee JY (2014) Reputation computation in social networks and its applications

9. Lee JY, Duan Y, Oh JC, Du W, Blair H, Wang L, Jin X (2011) Automatic reputation computation through document analysis: a social network approach. In: 2011 international conference on advances in social networks analysis and mining (ASONAM), pp 559-560. IEEE

10. Lee JY, Duan Y, Oh JC, Du W, Blair H, Wang L, Jin X (2012) Social network based reputation computation and document classification. J UCS 18(4):532-553

11. Lee JY, Lopatin K, Hussain R, Nawaz W (2017) Evolution of friendship: a case study of mobiclique. In: Proceedings of the computing frontiers conference. ACM, pp 267-270

12. Lee JY, Oh JC (2014) Estimating the degrees of neighboring nodes in online social networks. In: International conference on principles and practice of multi-agent systems. Springer, pp 42-56

13. Li Z (Lionel), Fang X, Sheng ORL (2015) A survey of link recommendation for social networks: methods, theoretical foundations, and future research directions. CoRR, abs/1511.01868

14. Solonets S, Drobny V, Rivera V, Lee JY (2017) Introducing ADegree: anonymisation of social networks through constraint programming, LNCS. Springer

15. Szczepanski PL, Barcz AS, Michalak TP, Rahwan T (2015) The game-theoretic interaction index on social networks with applications to link prediction and community detection. In: Proceedings of the twenty-fourth IJCAI 2015, Buenos Aires, Argentina, July 25-31, pp 638-644

16. Tigunova A, Lee JY, Nobari S (2015) Location prediction via social contents and behaviors: location-aware behavioral lda. In: 2015 IEEE international conference on data mining workshop (ICDMW). IEEE, pp 1131-1135

17. Wu L, Ge Y, Liu Q, Chen E, Long B, Huang Z (2016) Modeling users' preferences and social links in social networking services: a joint-evolving perspective. In: Proceedings of the thirtieth AAAI, Feb 12-17, Phoenix, AZ, USA, pp 279-286

18. Zhang J, Tang J, Li J, Liu Y, Xing C (2015) Who influenced you? predicting retweet via social influence locality. TKDD 9(3):25 